\title{
Staged reconstruction of a chronically infected large skull defect using free tissue transfer and a patient- specific polyetheretherketone implant
}

\author{
Seung Jin Moon ${ }^{1,2}$, \\ Hong Bae Jeon ${ }^{1,2}$, \\ Eui Hyun $\mathrm{Kim}^{3}$, \\ Dae Hyun Lew ${ }^{1,2}$, \\ Yong Oock Kim ${ }^{1,2}$ \\ Jong Won Hong ${ }^{1,2}$
}

${ }^{1}$ Department of Plastic and Reconstructive Surgery, ${ }^{2}$ Institute for Human Tissue Restoration, and ${ }^{3}$ Department of Neurosurgery, Yonsei University College of Medicine, Seoul, Korea
Reconstructions of extensive composite scalp and cranial defects are challenging due to high incidence of postoperative infection and reconstruction failure. In such cases, cranial reconstruction and vascularized soft tissue coverage are required. However, optimal reconstruction timing and material for cranioplasty are not yet determined. Herein, we present a large skull defect with a chronically infected wound that was not improved by repeated debridement and antibiotic treatment for 3 months. It was successfully treated with anterolateral thigh (ALT) free flap transfer for wound salvage and delayed cranioplasty with a patient-specific polyetheretherketone implant. To reduce infection risk, we performed the cranioplasty 1 year after the infection had resolved. In the meantime, depression of ALT flap at the skull defect site was observed, and the midline shift to the contralateral side was reported in a brain computed tomography (CT) scan, but no evidence of neurologic deterioration was found. After the surgery, sufficient cerebral expansion without noticeable dead-space was confirmed in a follow-up CT scan, and there was no complication over the 1-year follow-up period.

Keywords: Cranioplasty / Free tissue flap / Polyetheretherketone

\section{INTRODUCTION}

Composite scalp and cranial defects are not uncommon problems, especially in cases with infection or radiation. The cranial defects can cause problems in brain protection, aesthetic appearance, cerebral hydrodynamics, and neurologic function, thereby require cranioplasties [1,2]. Also, proper soft tissue coverage over the cranioplasty material is mandatory for a successful reconstruction. Despite many options for skull and scalp reconstruction, determining a cranioplasty material and soft tis-

\footnotetext{
Correspondence: Jong Won Hong

Department of Plastic and Reconstructive Surgery, Yonsei University College of

Medicine, 50-1 Yonsei-ro, Seodaemun-gu, Seoul 03722, Korea

E-mail: saturn@yuhs.ac

This study was supported by a faculty research grant of Yonsei University College of Medicine (grant No. 6-2016-0147).

Received July 7, 2020 / Revised August 31, 2020 / Accepted October 14, 2020
}

sue coverage method is challenging for a large complex defect on scalp and cranium [3]. A non-vascularized bone flap is inappropriate to be grafted in unhealthy soft tissue. A chimeric free flap, such as vascularized scapular bone and latissimus dorsi (LD) flap, can be utilized to reconstruct composite defects and has a high ability to resist infection and bone resorption [4]. However, since the available quantity of bone in a chimeric flap is limited, an alloplastic implant would be reasonable for a sizable defect. Furthermore, free soft tissue transfer for scalp reconstruction is necessary when defects are extensive that local or regional options are unavailable.

In alloplastic cranioplasty, one of the main complications is infection [5]. Therefore, when reconstructing composite defects with a history of infection, decisions on methods and timing of reconstruction should be made carefully. In this report, we describe a patient who underwent scalp reconstruction with free 
vascularized flap and delayed cranioplasty using a patient-specific polyetheretherketone (PEEK) implant for a massive cranial defect with a chronically infected wound which was not improved by repeated surgical debridement and antibiotic treatment for 3 months.

\section{CASE REPORT}

A 31-year-old male patient visited Severance Hospital for a scalp and skull defect and uncontrolled wound infection (Fig. 1). Two years earlier, the patient had a decompressive craniectomy for traumatic subarachnoid hemorrhage and intracranial hemorrhage due to a motorcycle accident. Subsequently, drainage and insertion of an external ventricular drain for a subdural abscess were performed. Cranioplasty with cryopreserved autologous bone graft was performed 6 months after the trauma. The patient continuously scratched the surgical site with his hand, which resulted in a subsequent scalp wound and infection. The infected bone graft was removed, and the soft tissue defect was repaired with local flap advancement. However, surgical site infection and scalp defect recurred, and it was not improved with continuous wound care, debridement, and antibiotic treatment for 3 months. The patient was admitted to our hospital for infection control and reconstruction. At the time of the admission, his level of consciousness was alert, but he was quadriplegic and showed a low score (19) on the Mini-Mental State Examination. Previously grafted artificial dura was exposed, and Klebsiella pneumonia, methicillin-resistant Staphylococcus aureus (MRSA), and Acinetobacter baumannii were identified in wound cultures.

Since resolution of the infection was a priority, removal of the

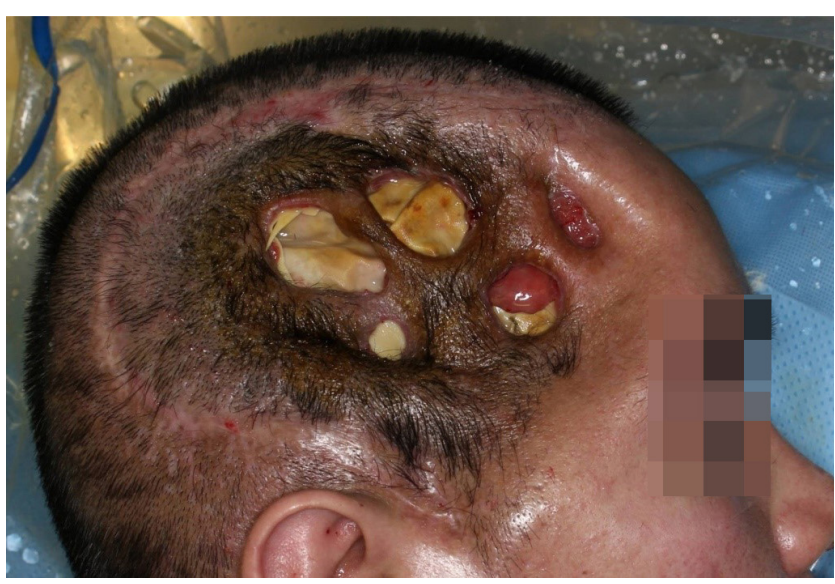

Fig. 1. Preoperative photograph of the skull and scalp defect on the right temporo-parietal area. The artificial dura is exposed. Klebsiella pneumoniae, methicillin-resistant Staphylococcus aureus, and Acinetobacter baumannii were identified in the wound culture. exposed artificial dura, extensive debridement including infected bone, massive irrigation, duroplasty with Biodesign dural graft (Cook Medical, Bloomington, IN, USA), and defect coverage with LD myocutaneous free flap and split-thickness skin graft were performed (Fig. 2). Considering the high risk of infection, cranioplasty was planned to be performed at least 1 year after confirming resolution of the infection. The size of the LD flap and its skin paddle was $17 \times 19 \mathrm{~cm}^{2}$ and $7 \times 19 \mathrm{~cm}^{2}$, respectively. The facial artery and vein on the right cheek were prepared as donor vessels, and anastomosis was performed.

Nine days after the surgery, the skin graft became unstable, and necrosis of the flap was observed. MRSA and A. baumannii were reported from pus-like fluid accumulated under the flap. A detachment of the LD flap, debridement, duroplasty, and $17 \times 19 \mathrm{~cm}^{2}$ sized anterolateral thigh (ALT) myocutaneous free flap transfer were performed, and the ALT flap was taken suc-
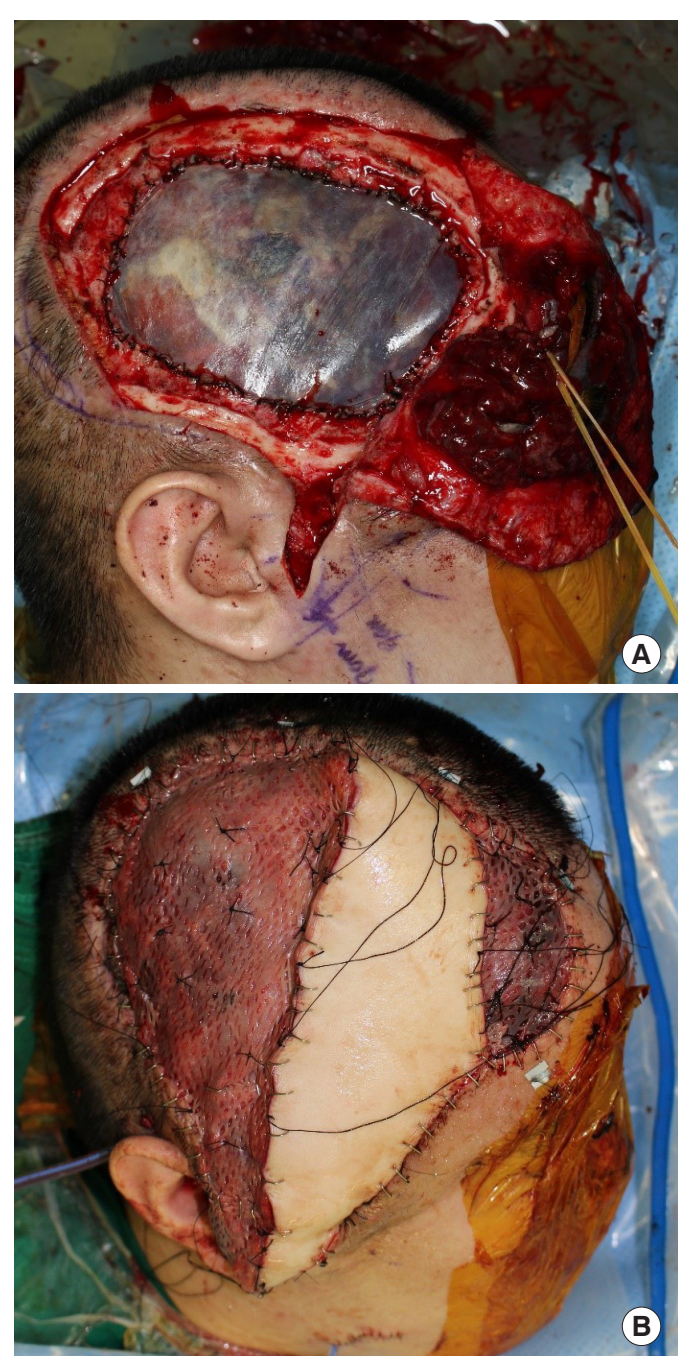

Fig. 2. (A) Intraoperative photograph after debridement and duroplasty. (B) The defect is covered with a latissimus dorsi myocutaneous free flap and split-thickness skin graft. 
cessfully. Vessel anastomosis was performed proximal to the same recipient vessels.

The reconstructed area initially had a convex shape due to a sufficient volume of the ALT flap. Six months after the second microsurgical surgery, flap sinking on the cranial defect area was observed (Fig. 3). However, since there was no evidence of neurologic deterioration, we decided to perform the cranial reconstruction following the originally planned schedule.

The cranioplasty was done with a patient-specific PEEK implant (Depuy Synthes; Oberdorf, Switzerland) at 1 year and 3 months form the second microsurgical reconstruction. The implant was designed with less curvature than of the contralateral side because we worried that the pedicle vessel would be damaged if the flap was stretched out too much. Furthermore, the implant was designed to match the irregular thickness of the re-

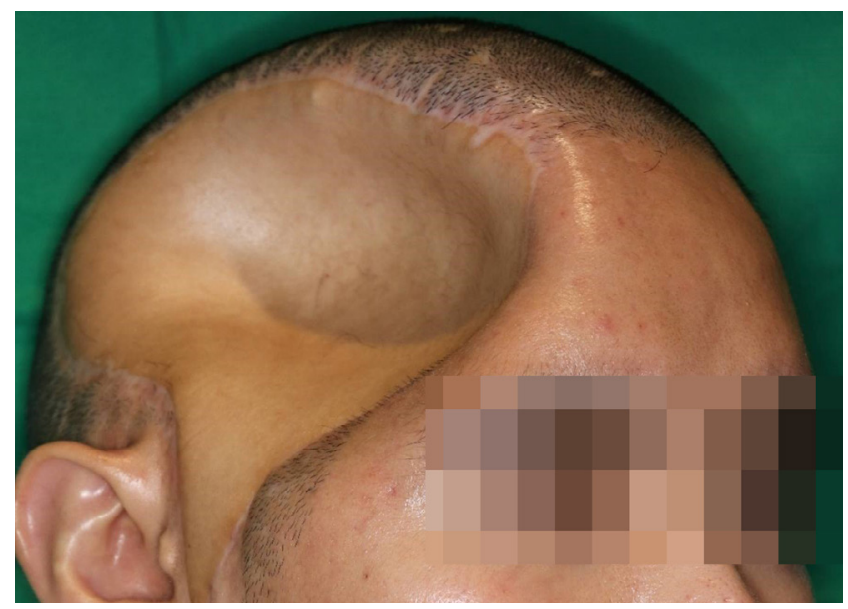

Fig. 3. Depression of the anterolateral thigh flap on the temporoparietal cranial defect.

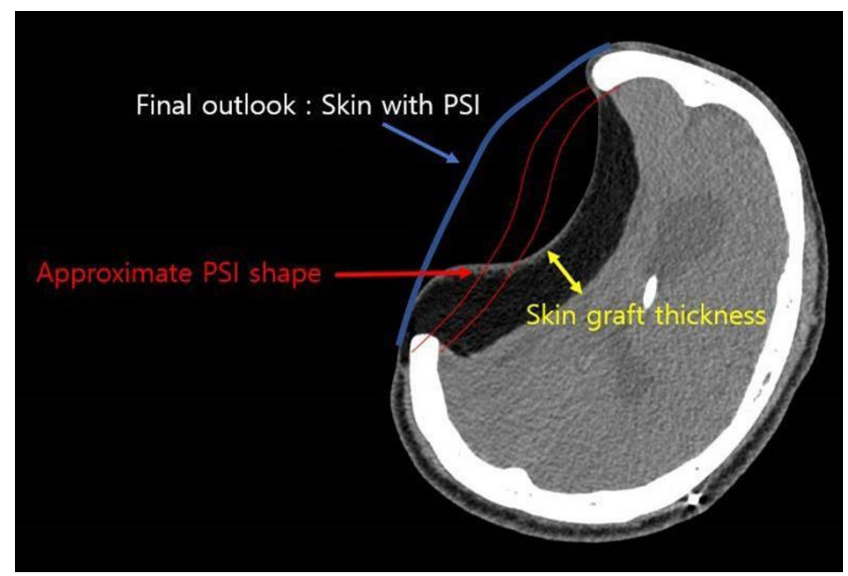

Fig. 4. Planning computed tomography image and design of the patient-specific implant (PSI). Shifting of brain parenchyma and decrease in ventricular size was observed. The irregular thickness of the reconstructed scalp flap was considered to achieve a smooth and concave head shape after cranioplasty. constructed scalp flap observed in the planning computed tomography (CT) scan to achieve a smooth and concave head shape considering (Fig. 4). During surgery, previously grafted ALT flap was successfully separated from the dura with careful protection of the flap pedicle. Pedicle identification and flap monitoring using Doppler were performed periodically. After the skull defect was fully exposed, bone margin debridement, dura tenting suture, and implant fixation with titanium hardware were performed (Fig. 5). Finally, the flap was sutured to the scalp flap in its original position with minimal tension. The depressed external contour was improved after the cranioplasty and the postoperative CT scan identified sufficient brain re-expansion and normalization of ventricle sizes (Fig. 6). There was no evidence of complications such as infection, wound dehiscence, or fluid collection over the 1-year follow-up period (Fig. 7).

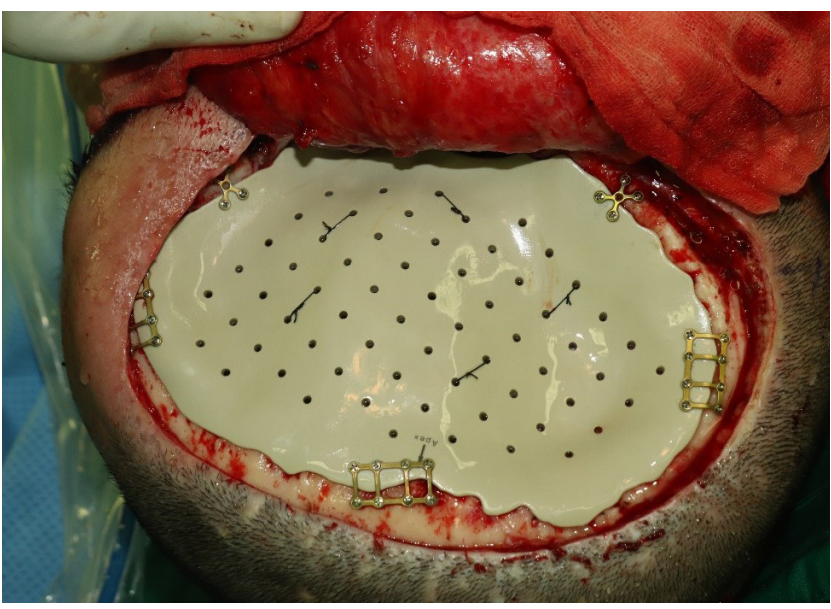

Fig. 5. Intraoperative photograph after dura tenting suture and fixation of the patient-specific polyetheretherketone implant.

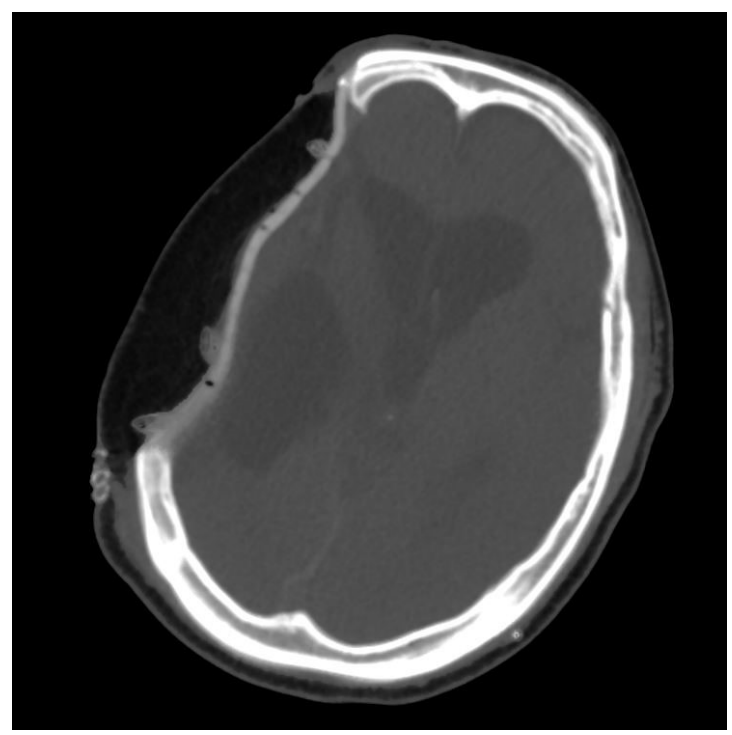

Fig. 6. Computed tomography scan at 1 week after the cranioplasty. 


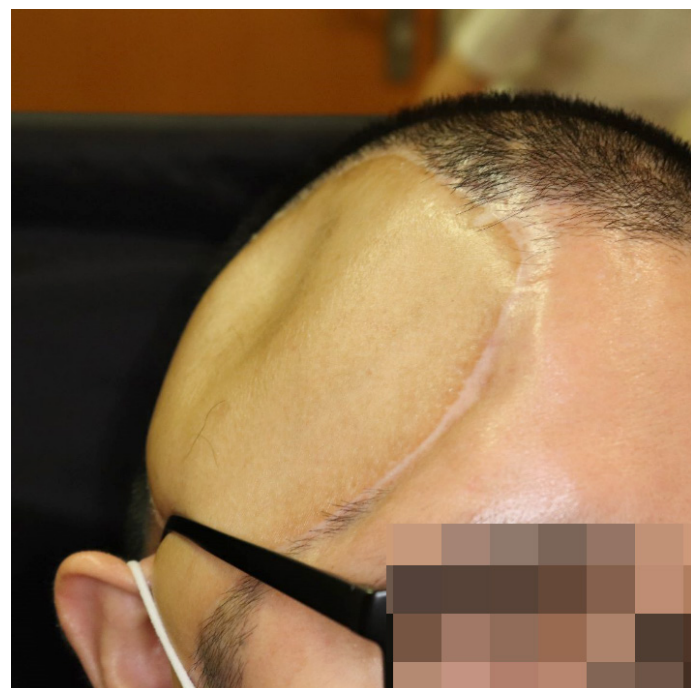

Fig. 7. Photograph of the patient at the 1-year follow-up.

\section{DISCUSSION}

When reconstructing complex scalp and cranial defects, careful consideration must be given to the selection of cranioplasty materials, soft tissue coverage methods, and timing of surgeries, regarding histories of prior surgery, infection, and adjuvant therapy on the defect area. Simultaneous reconstruction of both scalp and cranium may reduce a burden of repetitive general anesthesia and show quicker recovery. However, single-stage reconstruction may not be feasible in the cases of sizable and currently infected composite cranial defects which need wound salvage quickly.

In the present case, before the first microsurgical reconstruction, infection on the extensive defect was not improved with continuous intravenous antibiotics and surgical debridement, and exposure of artificial dura was observed. A LD flap was initially selected for scalp reconstruction because muscle flaps are relatively resistant to infection and can cover large areas with minimal donor site morbidity [6]. However, due to the flap failure, the second free flap transfer was performed with the ALT flap, which was harvested as a myocutaneous flap for the same reason as the LD flap.

Recent advances in computer-assisted design and computerassisted manufacturing technologies have enabled patient-specific implants based on titanium, poly-methyl-methacrylate (PMMA), and PEEK to reduce surgical time and pursue better cosmetic results [7,8]. A PEEK-based implant has several advantages over other cranioplasty materials. It is lighter than a titanium implant of the same volume, resistant to heat and radiation, and radiolucent. In addition, PEEK has stiffness and strength similar to bones, so it can provide sufficient brain pro- tection and be handled similarly to bones [9]. For example, modification of PEEK implants with burring during surgeries and use of conventional fixation hardware are possible. In a meta-analysis comparing with titanium mesh and autologous bone graft, PEEK-based implants showed a similar risk of complications, including infection, hematoma, exposure, and failure of reconstruction [10]. However, to our knowledge, there is no paper analyzing long-term results of more than 5 years after cranioplasty with a patient-specific PEEK implant. Infection may appear even after 1 year of alloplastic cranioplasty, and the average time interval between cranioplasty with titanium mesh and implant exposure occurrence was reported as 33.6 months [11]. Although no infection or exposure of the patient-specific PEEK implant was observed in this study, 1-year follow-up period after the cranioplasty may be not enough to confirm the safety of the implant. A large sample study with long-term follow-up is needed to elucidate effectiveness and reliability of PEEK implants.

There are several points to consider when designing a patientspecific implant. Since the skull has a round shape, the implant is usually designed in a convex shape, which is also intended to make space for brain parenchyma expansion [12]. However, the brain may not be expanded sufficiently in cases with histories of repeated surgeries or infection, which make fibrotic tissue on the defect area. Therefore, the final head shape on the defect was designed to be less convex than that on the contralateral side. Besides, it was necessary to prevent excessive tension on the previously grafted ALT free flap after implant reconstruction.

After implant reconstruction, brain expansion occurred sufficiently, and no dead-space was observed in the follow-up CT scan. Despite the gross improvement of the scalp depression, there was a mild irregularity of the final scalp contour. We expected that irregular thickness of the flap on the planning CT scan would be maintained after the cranioplasty. Nevertheless, soft tissue thickness on the defect area was nearly even on the postoperative CT scan, thereby revealing the implant's curvature. Infection is a significant complication when using allografts and can lead to reconstruction failure. Manson et al. [13] reported that the ideal time to reconstruct a skull defect of a frontal area with an alloplastic material is more than 1 year from the previous injury or infection. Kwiecien et al. [14] investigated the relationship between the timing of alloplastic cranioplasty after osteomyelitis and reinfection, finding that the reinfection rate was reduced by $10 \%$ for each month of cranioplasty delayed after removal of the infected bone. Although some reports demonstrated that early cranioplasty is feasible for patients with osteomyelitis [15,16], we decided to perform cranioplasty with the PEEK implant after confirming no evidence of 
infection for at least 1 year since the patient had the history of repeated infection for 2 years.

In conclusion, the present study suggests that early soft tissue coverage with free flap and delayed cranioplasty with a customized PEEK implant is an appropriate option to reconstruct extensive scalp and cranial defects with chronically infected wounds. Since a sinking skin flap on a skull defect may cause a shift of brain parenchyma, changes in cerebrospinal fluid hydrodynamics, and rarely, neurological dysfunction [17,18], regular follow-up with brain CT and neurological examination is necessary during the time between the soft tissue coverage and the cranioplasty.

\section{NOTES}

\section{Conflict of interest}

No potential conflict of interest relevant to this article was reported.

\section{Ethical approval}

The study was approved by the Institutional Review Board of Severance Hospital, Yonsei University Health System (IRB No. 4-2020-0050) and performed in accordance with the principles of the Declaration of Helsinki. Written informed consent was obtained.

\section{Patient consent}

The patient provided written informed consent for the publication and the use of his images.

\section{ORCID}

Seung Jin Moon

Hong Bae Jeon

Eui Hyun Kim

Dae Hyun Lew

Yong Oock Kim

Jong Won Hong https://orcid.org/0000-0003-1980-0270 https://orcid.org/0000-0003-2412-4537 https://orcid.org/0000-0002-2523-7122 https://orcid.org/0000-0002-2625-5664 https://orcid.org/0000-0002-3756-4809 https://orcid.org/0000-0002-7762-0940

\section{REFERENCES}

1. Honeybul S. Neurological susceptibility to a skull defect. Surg Neurol Int 2014;5:83.

2. Dujovny M, Fernandez P, Alperin N, Betz W, Misra M, Mafee M. Post-cranioplasty cerebrospinal fluid hydrodynamic changes: magnetic resonance imaging quantitative analysis. Neurol Res 1997;19:311-6.

3. Aydin S, Kucukyuruk B, Abuzayed B, Aydin S, Sanus GZ. Cranioplasty: review of materials and techniques. J Neurosci Rural
Pract 2011;2:162-7.

4. Lee JC, Kleiber GM, Pelletier AT, Reid RR, Gottlieb LJ. Autologous immediate cranioplasty with vascularized bone in highrisk composite cranial defects. Plast Reconstr Surg 2013;132: 967-75.

5. Reddy S, Khalifian S, Flores JM, Bellamy J, Manson PN, Rodriguez ED, et al. Clinical outcomes in cranioplasty: risk factors and choice of reconstructive material. Plast Reconstr Surg 2014;133:864-73.

6. Lutz BS, Wei FC, Chen HC, Lin CH, Wei CY. Reconstruction of scalp defects with free flaps in 30 cases. Br J Plast Surg 1998; 51:186-90.

7. Bonda DJ, Manjila S, Selman WR, Dean D. The recent revolution in the design and manufacture of cranial implants: modern advancements and future directions. Neurosurgery 2015; 77:814-24.

8. Choi JW, Kim N. Clinical application of three-dimensional printing technology in craniofacial plastic surgery. Arch Plast Surg 2015;42:267-77.

9. Persson J, Helgason B, Engqvist H, Ferguson SJ, Persson C. Stiffness and strength of cranioplastic implant systems in comparison to cranial bone. J Craniomaxillofac Surg 2018;46:41823.

10. Punchak M, Chung LK, Lagman C, Bui TT, Lazareff J, Rezzadeh K, et al. Outcomes following polyetheretherketone (PEEK) cranioplasty: systematic review and meta-analysis. J Clin Neurosci 2017;41:30-5.

11. Thien A, King NK, Ang BT, Wang E, Ng I. Comparison of polyetheretherketone and titanium cranioplasty after decompressive craniectomy. World Neurosurg 2015;83:176-80.

12. Fodstad H, Love JA, Ekstedt J, Friden H, Liliequist B. Effect of cranioplasty on cerebrospinal fluid hydrodynamics in patients with the syndrome of the trephined. Acta Neurochir (Wien) 1984;70:21-30.

13. Manson PN, Crawley WA, Hoopes JE. Frontal cranioplasty: risk factors and choice of cranial vault reconstructive material. Plast Reconstr Surg 1986;77:888-904.

14. Kwiecien GJ, Aliotta R, Bassiri Gharb B, Gastman B, Zins JE. The timing of alloplastic cranioplasty in the setting of previous osteomyelitis. Plast Reconstr Surg 2019;143:853-61.

15. Wind JJ, Ohaegbulam C, Iwamoto FM, Black PM, Park JK. Immediate titanium mesh cranioplasty for treatment of postcraniotomy infections. World Neurosurg 2013;79:207.

16. Lopez J, Zhong SS, Sankey EW, Swanson EW, Susarla H, JusueTorres I, et al. Time interval reduction for delayed implantbased cranioplasty reconstruction in the setting of previous bone flap osteomyelitis. Plast Reconstr Surg 2016;137:394e$404 \mathrm{e}$. 
17. Annan M, de Toffol B, Hommet C, Mondon K. Sinking skin flap syndrome (or syndrome of the trephined): a review. $\mathrm{Br} \mathrm{J}$ Neurosurg 2015;29:314-8.
18. Ashayeri K, Jackson EM, Huang J, Brem H, Gordon CR. Syndrome of the trephined: a systematic review. Neurosurgery 2016;79:525-34. 